\title{
Adaptación, validez y fiabilidad del inventario ansiedad rasgo- estado para adultos de la ciudad de Trujillo
}

\author{
Adaptation, validity and reliability of the trait anxiety inventory - state for \\ adults of the city of Trujillo
}

\author{
Lili Claudina Bazán Izquierdo ${ }^{1}$ \\ Ministerio de la Mujer y Poblaciones Vulnerables
}

Recibido: $30-04-21$

Aceptado: $17-05-21$

Publicado: $18-06-21$

\section{Resumen}

El Inventario de Ansiedad Rasgo-Estado (IDARE) ha demostrado apropiados niveles de validez y fiabilidad para su uso en diferentes países, sin embargo, aún no se presentan evidencias para su uso en adultos de la ciudad de Trujillo. El objetivo del estudio fue adaptar y validar el IDARE en una muestra de adultos. La muestra se conformó por 120 padres (96 mujeres y 24 hombres) con una edad media de 35.18 años. Los resultados indicaron que el instrumento presenta garantías de validez basadas en el contenido y en la revisión de expertos sus ítems manifestaron ser claros, coherentes y relevantes $\left(\mathrm{V}_{\text {Aiken }}>.70, \mathrm{p}<.05\right)$. Una segunda versión del IDARE, con 37 ítems ( 3 menos en la dimensión ansiedad rasgo), reportó mejores índices de ajuste $\left(\mathrm{x}^{2} / \mathrm{gl}=1.74 ;\right.$ RSMEA $=.080($ IC $95 \%=.072-.088)$; SRMR $=.096$; CFI $=.988$ y TLI $=.987)$, con ítems pertinentes en la medida de cada dimensión $(\lambda>.40)$ y fiabilidad aceptable en ambas dimensiones, ansiedad estado y ansiedad rasgo $(\omega>.70)$. Estos resultados demuestran que el IDARE puede ser aplicado en la población estudiada con ligeras modificaciones de la versión original.

Palabras clave: Ansiedad estado; ansiedad rasgo; evidencias de validez; confiabilidad.

\begin{abstract}
The State-Trait Anxiety Inventory (IDARE) has demonstrated appropriate levels of validity and reliability for its use in different countries, however, evidence for its use in adults in the city of Trujillo has not yet been presented. The aim of the study was to adapt and validate the IDARE in a sample of adults. The sample consisted of 120 parents (96 women and 24 men) with a mean age of 35.18 years. The results indicated that the instrument presents validity guarantees based on the content and in the expert review, its items showed to be clear, coherent and relevant (VAiken $>.70, p<.05$ ). A second version of the IDARE, with 37 items ( 3 less in the trait anxiety dimension), reported better fit indices ( $\mathrm{x} 2 / \mathrm{gl}=1.74$; RSMEA $=$
\end{abstract}

1 Psicóloga en el Centro de Emergencia Mujer - Ministerio de la Mujer y Poblaciones Vulnerables, Lima, Perú. Autor para correspondencia: lilibazan879@gmail.com, lili.bazan@unmsm.edu.pe ORCID: https://orcid.org/0000-0002-9974-4784

(C) Los autores. Este artículo es publicado por la Revista de Investigación en Psicología de la Facultad de Psicología, Universidad Nacional Mayor de San Marcos. Este es un artículo de acceso abierto, distribuido bajo los términos de la licencia Creative Commons Atribución 4.0 Internacional (CC BY 4.0) [https://creativecommons.org/licenses/by/4.0/deed.es] que permite el uso, distribución y reproducción en cualquier medio, siempre que la obra original sea debidamente citada de su fuente original. 
$.080(95 \% \mathrm{CI}=.072-.088) ; \mathrm{SRMR}=.096 ; \mathrm{CFI}=.988$ and $\mathrm{TLI}=.987)$, with relevant items in the measure of each dimension $(\lambda>.40)$ and acceptable reliability in both dimensions, state anxiety and trait anxiety $(\omega>.70)$. These results show that IDARE can be applied in the population studied with slight modifications from the original version.

Keywords: State anxiety, trait anxiety, evidence of validity, reliability, STAI.

\section{INTRODUCCIÓN}

En los últimos años, los índices de ansiedad han ido en aumento (Fernández \& Espinoza, 2019), y su incidencia no distingue estrato sociocultural, convirtiéndose en un serio problema de salud mental. Por ello, conocer los niveles de ansiedad ayuda a los profesionales a identificar cómo se encuentra la persona y de qué manera puede ayudarla.

La ansiedad es considerada uno de los desórdenes psiquiátricos con una elevada prevalencia en el mundo, ubicándose en la posición octava dentro de los trastornos que padece la población (Bouayed et al., 2009). Entre 1990 y 2013, la ansiedad, junto a la depresión, constituyó el trastorno mental con mayor incremento a nivel mundial (Organización Mundial de la Salud [OMS], 2020). Para el 2017 se reportaron a 264 millones de sujetos con el diagnóstico de trastorno ansioso (OMS, 2019) lo cual equivale a 1 de cada 5 personas en todo el mundo.

En el Perú, el Instituto Nacional de Salud Mental (INSM) indicó que, en el año 2012, en Lima, la prevalencia de pacientes adultos con trastornos de ansiedad alcanzaba el 3.3\%; mientras que el 3.0\% de adolescentes reportaba ansiedad generalizada; y el $2.6 \%$, fobia social. En el adulto mayor, la ansiedad alcanzaba una prevalencia del 3.8\% (INSM, 2013). Para el 2018, se registraron datos de otras ciudades, por ejemplo, el Ministerio de Salud (MINSA) señala que en Huancavelica la prevalencia alcanzó el 2.4\% y en Tacna fue de 10.1\% (Broca, 2019). Díaz (2018) refiere que, en La Libertad, la prevalencia fue del $22 \%$ de la población. Como se puede observar, el trastorno de ansiedad ha aumentado considerablemente; presentándose, incluso, a más corta edad.

Cabe precisar que la ansiedad es uno de los componentes principales de todo trastorno psicológico y de somatización, la cual constituye un estado intrínseco consciente explicado como una emoción, un afecto o un sentimiento vinculado a un temor indefinido (Zych, 2011). Se considera una experiencia adaptativa para enfrentar diversas circunstancias (Rojas, 2014) o un estado de presión donde la persona es consciente de que se enfrenta a un peligro y reacciona de manera fisiológica.

Para Spielberger et al. (1970), la ansiedad puede ser vista desde de dos perspectivas: como estado y como rasgo. Desde la primera perspectiva, la ansiedad es un estado o condición emocional transitoria del organismo humano que varía en 
intensidad y fluctúa con el tiempo; además, se entiende que resulta del momento, es decir, de la situación y circunstancia (García-Batista et al., 2015). Esta condición se caracteriza por sentimientos subjetivos de tensión y aprehensión, percibidos conscientemente, y la activación del sistema nervioso autónomo (Spielberger \& Díaz- Guerrero, 1975).

Desde la segunda perspectiva, las respuestas afectivas ansiosas surgen independientemente del momento, pero influenciadas por aspectos de la personalidad del propio sujeto (García-Batista et al., 2015). Se basa en las diferencias individuales relativamente estables que conforman una propensión a la ansiedad, es decir, en la existencia de rasgos que constituyen disposiciones personales para percibir una variedad de situaciones de estímulo como peligrosas o amenazantes, así como la respuesta a tales amenazas con reacciones de estado (Guevara - Cordero et al., 2019).

La teoría se ocupa de aclarar las propiedades del estado y del rasgo como construcciones psicológicas, y de especificar las características de las condiciones de estímulo estresante que evocan niveles diferenciales del estado en personas que difieren en el rasgo (Spielberger et al., 1970). La teoría también reconoce la centralidad de la evaluación cognitiva en la evocación de un estado de ansiedad, y la importancia de los procesos cognitivos y motores (mecanismos de defensa) que sirven para eliminar o educar los estados de ansiedad (Spielberger, 1975).

En lo que se refiere al problema de la ansiedad es necesario contar con herramientas que sirvan de respaldo a la evaluación y diagnóstico que realizan los profesionales psicólogos y psiquiatras. Esto permitiría que las mediciones se efectúen con mayor precisión y objetividad y, como consecuencia, se puedan generar intervenciones más efectivas.

Al respecto, el Inventario de Ansiedad: Estado-Rasgo (de nombre original State-Trait Anxiety Inventory [STAI]), elaborado por Spielberger, Gorsuch \& Lushene (1970), adaptado aproximadamente 60 veces (Buela-Casal et al., 2011) y usado en cerca de 14 mil investigaciones (Guillen-Riquelme \& Buela-Casal, 2015). En los estudios realizados ha demostrado ser un instrumento con medidas consistentes y estables, que cumple con evidencias de validez basadas en la relación con otros test, tanto en España como en Latinoamérica (García-Batista et al.,2017).

El STAI fue adaptado para la población latinoamericana por Spielberger \& Díaz-Guerrero en 1975, renombrándose como Inventario de Ansiedad: Rasgo-Estado (IDARE). Respecto al análisis psicométrico como versión IDARE, las investigaciones han arrojado resultados variados.

Al revisarse en padres de niños en terapia intensiva, las cargas de una exploración de los factores reportaron al ítem 34 "Trato de sacarle el cuerpo a las crisis y dificultades" con carga factorial baja; pero fue conservado por no afectar a la confiabilidad del instrumento, cuyos valores fueron de .85 AE y .89 AR (Rojas-Carrasco, 
2010). Luego, en población universitaria, se identificó un comportamiento similar en el ítem 34, carga factorial baja, sumándosele el ítem 26 ("Me siento descansado") cuando la estructura obtenía índices de ajuste (SRMR $=.72$; NFI y RFI $=.93$ ) y confiabilidad de .90 en AE y .87 AR (Espíritu, 2018).

Esta es la razón que despierta el interés por realizar la adaptación del IDARE para la población de Trujillo, Perú, antes de utilizarse como herramienta de medida de la ansiedad. Para el estudio se planteó como objetivo adaptar el IDARE y analizar sus fuentes de validez en población adulta de Trujillo. Asimismo, es un aporte importante para garantizar científicamente el uso del instrumento en adultos de Trujillo, Perú.

\section{MÉTODO}

La investigación se desarrolló a partir del diseño de estudio clasificado como instrumental. Estos estudios, orientan en la revisión de las propiedades de medida de un test o prueba psicológica, nuevos o ya existentes a partir de determinados estándares (Ato et al., 2013).

\section{Participantes}

La población considerada para la investigación es la población diana, es decir, una población grande y de difícil acceso (Ventura-León, 2017). Tal población es de adultos de la ciudad de Trujillo. La selección de participantes se realizó a través de un muestreo impulsado por los encuestados (Heckathorn, 2007), cuyo diseño es aplicable cuando se desconoce la población de acceso y se mueve a través de plataformas online (cuestionarios virtuales) para lo cual se siguió el siguiente patrón: se recolectó un número determinado de participantes (adultos) y luego se expandió la incorporación de nuevos participantes invitados por el grupo inicial y se repitió el proceso hasta superar una cantidad mínima de participantes requeridos

La muestra estuvo conformada por 120 adultos de ambos sexos (96 mujeres y 24 hombres), de edades comprendidas entre los 24 y 51 años $(M=35.18$ años, $D T$ $=6.33$ ) a los cuales se accedió por medio de formularios de Google, dada las crisis de pandemias que se atravesaba en el mundo durante el periodo en que se llevó a cabo el estudio; año 2020.

\section{Instrumento}

Para realizar el presente estudio se aplicó una ficha sociodemográfica para la caracterización de los adultos. Este cuestionario estuvo conformado por nueve preguntas cerradas: sexo, edad, lugar de residencia, grado de instrucción, ocupación y diagnóstico sobre ansiedad. 
También, se aplicó el inventario de Ansiedad Rasgo - Estado (IDARE) versión adaptada por Spielberger y Díaz-Guerrero (2002), publicada en la editorial El Manual Moderno (México). Esta adaptación cuenta con 40 ítems (20 ítems por escala), con respuestas de formato tipo Likert. Su aplicación puede ser individual o colectiva, sin límite de tiempo y se administra en un tiempo que va de 12 a 20 minutos, según el grado de instrucción del evaluado (Spielberger y Díaz-Guerrero, 1975).

\section{Procedimiento}

La investigación se realizó en tres etapas: a) adaptación del instrumento mediante la evaluación de expertos. Para lo cual se tomó contacto con nueve psicólogos clínicos especialistas en ansiedad, revisaron el inventario de manera independiente y valoraron cualitativamente el nivel de claridad, coherencia y relevancia del instrumento. El resultado de esta evaluación indicó las siguientes modificaciones: ítem 4, se modificó "me siento contrariado" por "me siento malhumorado" y el ítem 15 , se cambió "me siento reposado" por "Estoy experimentando una sensación de calma". b) posteriormente a través de las redes sociales se contactó a adultos que cuenten con las características de investigación solicitada, se explicó el objetivo de la investigación y se envió el link donde se encontraba el consentimiento informado, la ficha sociodemográfica y el inventario a evaluar. c) Finalmente se evaluó la validez y confiabilidad del inventario a través de variables latentes.

\section{Análisis de datos}

El análisis de la información se realizó apoyados en los siguientes softwares estadísticos: Excel 2019, Visual Basic y R Studio. Los métodos utilizados, de acuerdo a las evidencias de validez, fue el coeficiente $\mathrm{V}$ de Aiken, con sus respectivos intervalos de confianza ( $95 \%$ confianza) para responder a la evidencia de validez basada en el contenido. El criterio para valorar su cumplimiento señalaba que el límite inferior del coeficiente V debía tener un mínimo de $.70($ Vo $=0.70)$ (Charter, 2003).

Luego, respondiendo a la evidencia de validez basada en la estructura interna del inventario, se utilizó la técnica de análisis factorial confirmatorio, a partir del método Mínimos Cuadrados Ponderados Robustos, WLSMV, por sus iniciales en inglés (Brown, 2006). Los índices de ajuste seleccionados para responder a esta evidencia fueron la combinación Raíz Cuadrada Media del Error de Aproximación (RSMEA), Raíz del Residuo Cuadrático Estandarizado (S-RMR), Índice de Ajuste Comparativo (CFI) e Índice de Tuker-Lewis (TLI), debido a que entre la diversidad de índices han demostrado mayor resistencia ante la influencia de factores externos. Los criterios para su valoración como aceptables fueron: superior a .95 para TLI y CFI (Hu \& Bentler, 1999), con valores entre a .05 y .08 para RSMEA (García, 2011; Browne \& Cudeck) y entre .05 y 10 para SRMR (García, 2011; Hu \& Bentler, 1995).

La confiabilidad se estimó usando el coeficiente omega ( $\omega)$. En los últimos años, este coeficiente ha demostrado un mayor grado de estabilidad en su cálculo. 
Se estima utilizando las cargas factoriales que se obtienen en el análisis factorial y para su aceptación, se tomó en cuenta el valor de la consistencia, que se encuentra en un intervalo aceptable, con valores entre .70 y .90 (Campo-Arias \& Oviedo, 2008).

\section{RESULTADOS}

\section{Evidencia de validez de contenido}

El IDARE fue sometido a una revisión de expertos para verificar su validez referente al contenido. Para el caso de ambas escalas de ansiedad, se contó con la participación de $\mathrm{N}$ jueces $=9$. Los 20 ítems de la escala Ansiedad Estado [AE] reportaron coeficientes $\mathrm{V}_{\text {Aiken }}$ desde .81 a 1 en el criterio de claridad y coherencia; y desde .78 a .96 en el criterio de relevancia (Ver tabla 1). Valores que, de acuerdo con el criterio supuesto por Escurra (1988), resultaría estadísticamente significativo ( $p<.05$ en coeficientes V de Aiken superiores a .77 con 9 jueces). En consecuencia, se consideró que los ítems cumplen los tres criterios estudiados, es decir, los ítems son claros, coherentes y relevantes.

\section{Tabla 1}

Coeficientes V de Aiken de los items del IDARE, para la escala Ansiedad-Estado.

\begin{tabular}{|c|c|c|c|c|c|c|}
\hline \multirow{3}{*}{ ítem } & \multicolumn{6}{|c|}{ Revisión de expertos } \\
\hline & \multicolumn{2}{|c|}{ Claridad } & \multicolumn{2}{|c|}{ Coherencia } & \multicolumn{2}{|c|}{ Relevancia } \\
\hline & $\mathbf{V}$ & IC $95 \%$ & $\mathbf{v}$ & IC $95 \%$ & $\mathbf{V}$ & IC $95 \%$ \\
\hline 1 & 1.00 & {$[.88-1.0]$} & 0.96 & {$[.82-.99]$} & 0.93 & {$[.77-.98]$} \\
\hline 2 & 0.89 & {$[.72-.96]$} & 0.89 & {$[.72-.96]$} & 0.81 & {$[.63-.92]$} \\
\hline 3 & 1.00 & {$[.88-1.0]$} & 0.96 & {$[.82-.99]$} & 0.96 & {$[.82-.99]$} \\
\hline 4 & 1.00 & {$[.88-1.0]$} & 0.93 & {$[.77-.98]$} & 0.93 & {$[.77-.98]$} \\
\hline 5 & 0.96 & {$[.82-.99]$} & 0.93 & {$[.77-.98]$} & 0.93 & {$[.77-.98]$} \\
\hline 6 & 0.96 & {$[.82-.99]$} & 1.00 & {$[.88-1.0]$} & 0.93 & {$[.77-.98]$} \\
\hline 7 & 0.96 & {$[.82-.99]$} & 0.96 & {$[.82-.99]$} & 0.96 & {$[.82-.99]$} \\
\hline 8 & 1.00 & {$[.88-1.0]$} & 0.93 & {$[.77-.98]$} & 0.89 & {$[.72-.96]$} \\
\hline 9 & 1.00 & {$[.88-1.0]$} & 1.00 & {$[.88-1.0]$} & 0.89 & {$[.72-.96]$} \\
\hline 10 & 0.93 & {$[.77-.98]$} & 0.85 & {$[.68-.94]$} & 0.81 & {$[.63-.92]$} \\
\hline 11 & 1.00 & {$[.88-1.0]$} & 0.93 & {$[.77-.98]$} & 0.89 & {$[.72-.96]$} \\
\hline 12 & 0.89 & {$[.72-.96]$} & 0.93 & {$[.77-.98]$} & 0.89 & {$[.72-.96]$} \\
\hline 13 & 1.00 & {$[.88-1.0]$} & 0.96 & {$[.82-.99]$} & 0.96 & {$[.82-.99]$} \\
\hline 14 & 0.85 & {$[.68-.94]$} & 0.85 & {$[.68-.94]$} & 0.85 & {$[.68-.94]$} \\
\hline 15 & 0.93 & {$[.77-.98]$} & 0.93 & {$[.77-.98]$} & 0.81 & {$[.63-.92]$} \\
\hline 16 & 0.96 & {$[.82-.99]$} & 0.81 & {$[.63-.92]$} & 0.81 & {$[.63-.92]$} \\
\hline 17 & 1.00 & {$[.88-1.0]$} & 1.00 & {$[.88-1.0]$} & 0.89 & {$[.72-.96]$} \\
\hline 18 & 0.81 & {$[.63-.917]$} & 0.85 & {$[.68-.94]$} & 0.78 & {$[.59-.89]$} \\
\hline 19 & 1.00 & {$[.88-1.0]$} & 0.89 & {$[.72-.96]$} & 0.85 & {$[.68-.94]$} \\
\hline 20 & 0.89 & {$[.72-.96]$} & 0.81 & {$[.63-.92]$} & 0.78 & {$[.59-.89]$} \\
\hline
\end{tabular}

Nota: V: Coeficiente de validez de contenido V de Aiken 
Tabla 2

Coeficientes V de Aiken de los ítems del IDARE, para la escala Ansiedad-Rasgo.

\begin{tabular}{|c|c|c|c|c|c|c|}
\hline \multirow{3}{*}{ ítem } & \multicolumn{6}{|c|}{ Revisión de expertos } \\
\hline & \multicolumn{2}{|c|}{ Claridad } & \multicolumn{2}{|c|}{ Coherencia } & \multicolumn{2}{|c|}{ Relevancia } \\
\hline & $\mathbf{V}$ & IC $95 \%$ & $\mathbf{V}$ & IC $95 \%$ & $\mathbf{V}$ & IC $95 \%$ \\
\hline 21 & 0.96 & {$[.82-.99]$} & 0.85 & {$[.68-.94]$} & 0.85 & {$[.68-.94]$} \\
\hline 22 & 0.96 & {$[.82-.99]$} & 0.85 & {$[.68-.94]$} & 0.93 & {$[.77-.98]$} \\
\hline 23 & 1.00 & {$[.88-1.0]$} & 0.93 & {$[.77-.98]$} & 0.93 & {$[.77-.98]$} \\
\hline 24 & 1.00 & {$[.88-1.0]$} & 0.89 & {$[.72-.96]$} & 0.96 & {$[.82-.99]$} \\
\hline 25 & 1.00 & {$[.88-1.0]$} & 0.93 & {$[.77-.98]$} & 1.00 & {$[.88-1.0]$} \\
\hline 26 & 1.00 & {$[.88-1.0]$} & 0.89 & {$[.72-.96]$} & 0.85 & {$[.68-.94]$} \\
\hline 27 & 0.85 & {$[.68-.94]$} & 0.89 & {$[.72-.96]$} & 0.85 & {$[.68-.94]$} \\
\hline 28 & 0.93 & {$[.77-.98]$} & 0.93 & {$[.77-.98]$} & 0.93 & {$[.77-.98]$} \\
\hline 29 & 0.96 & {$[.82-.99]$} & 0.96 & {$[.82-.99]$} & 0.96 & {$[.82-.99]$} \\
\hline 30 & 1.00 & {$[.88-1.0]$} & 0.85 & {$[.68-.94]$} & 0.89 & {$[.72-.96]$} \\
\hline 31 & 0.89 & {$[.72-.96]$} & 0.89 & {$[.72-.96]$} & 0.85 & {$[.68-.94]$} \\
\hline 32 & 1.00 & {$[.88-1.0]$} & 0.93 & {$[.77-.98]$} & 0.89 & {$[.72-.96]$} \\
\hline 33 & 0.93 & {$[.77-.98]$} & 0.74 & {$[.55-.87]$} & 0.81 & {$[.63-.92]$} \\
\hline 34 & 1.00 & {$[.88-1.0]$} & 0.96 & {$[.82-.99]$} & 0.96 & {$[.82-.99]$} \\
\hline 35 & 0.93 & {$[.77-.98]$} & 0.85 & {$[.68-.94]$} & 0.85 & {$[.68-.94]$} \\
\hline 36 & 0.93 & {$[.77-.98]$} & 0.70 & {$[.52-.84]$} & 0.74 & {$[.55-.87]$} \\
\hline 37 & 0.93 & {$[.77-.98]$} & 1.00 & {$[.88-1.0]$} & 1.00 & {$[.88-1.0]$} \\
\hline 38 & 1.00 & {$[.88-1.0]$} & 0.93 & {$[.77-.98]$} & 0.93 & {$[.77-.98]$} \\
\hline 39 & 0.89 & {$[.72-.96]$} & 0.81 & {$[.63-.92]$} & 0.78 & {$[.59-.89]$} \\
\hline 40 & 1.00 & {$[.88-1.0]$} & 0.96 & {$[.82-.99]$} & 1.00 & {$[.88-1.0]$} \\
\hline
\end{tabular}

Nota: V: Coeficiente de validez de contenido V de Aiken

Por su parte, en los 20 ítems que corresponden a la escala de Ansiedad Rasgo [AR] (Ver tabla 2) se reportaron valores que van de .85 a 1.00 en claridad, de .70 a 1.00 en coherencia; y, de .74 a 1.00 en relevancia (Ver tabla 2). En este caso, el ítem 33 ["Me siento seguro (a)"] en coherencia y el ítem 36 ["Me siento satisfecho (a)"] en coherencia y relevancia; alcanzaron valores V por debajo del criterio sugerido Escurra (1988, $\mathrm{V}_{\text {Aiken }}>$.77). Pero, al explorar otros criterios de aceptación, se encontró la propuesta de Cicchetti (1994), quien sugiere valorar la aceptación a partir de la estimación de sus intervalos de confianza [IC], por medio del cual se aceptará un ítem como válido, cuando el límite inferior del intervalo de confianza sea mayor a $.50\left(\mathrm{~V}_{0} \geq .50\right)$. Entonces, al estimar el IC en coherencia, el ítem 33 obtuvo $\mathrm{V}_{\text {Aiken }}=.74$ (IC $95 \% .55-.87$ ) y el ítem 36 obtuvo $\mathrm{V}_{\text {Aiken }}=.70(\mathrm{IC} 95 \% .52-.84)$; en tanto, la relevancia el ítem 36 obtuvo $\mathrm{V}_{\text {Aiken }}$ $=.74$ (IC $95 \% .55$ - .87). Los valores superaron el mínimo de referencia, por lo cual se los consideró como válidos. 


\section{Evidencia de validez de estructura y fiabilidad}

Con las evidencias de validez del contenido, se procedió a revisar la estructura del IDARE. Este modelo presenta dos dimensiones: Dimensión de ansiedad-rasgo y dimensión de ansiedad-estado; cada una compuesta por 20 reactivos (AR del ítem 1 al 20; AE del ítem 21 al 40). El método aplicado para obtener las medidas de estructura fue el de Mínimos Cuadrados no Ponderados, cuyos resultados sobre el ajuste (Ver tabla 3) fueron de: $\mathrm{x}^{2}{ }_{(739)}=12.73 .98$, con una ratio de verosimilitud de 1.72; RSMEA $=.079$ (IC 95\% $=.072-.087)$; SRMR $=.100 ;$ CFI $=.986$ y TLI $=.985$. Estos valores demuestran el cumplimiento del ajuste por parte del modelo empírico y el modelo teórico hipotetizado con la distribución de ítems señalado al inicio del párrafo.

Tabla 3

Índices de bondad de ajuste

\begin{tabular}{cccccccc}
\hline Modelo & $\mathbf{X}^{2}$ & gl & $\mathbf{X}^{2} / \mathbf{g l}$ & RSMEA (IC90\%) & SRMR & CFI & TLI \\
\hline M1 & 1273.98 & 739 & 1.72 & $.079(.072-.087)$ & 0.100 & 0.986 & 0.985 \\
M2 & 1091.23 & 628 & 1.74 & $.080(.072-.088)$ & 0.096 & 0.988 & 0.987 \\
\hline
\end{tabular}

Nota: M1: Modelo original 40 ítems; M2: Modelo restructurado con 37 ítems; $\mathrm{x}^{2}$ : Chi cuadrado; gl: grados de libertad; $\mathrm{x}^{2} / \mathrm{gl}$ : ratio de verosimilitud; RSMEA: Error cuadrático medio de aproximación por grado de libertad; SRMR: raíz cuadrada de la media de residuos cuadrados; CFI: Índice de ajuste comparativo; TLI: Índice de Tukey-Lewis.

Los ítems fueron pertinentes para el factor al que estaban consignados, con mínimas excepciones. Sobre el factor AE, los 20 ítems alcanzaron cargas factoriales de .71 (item 3 "Estoy tenso (a)") a .90 (ítem 20 "Me siento bien") lo cual demostraría su representatividad al factor con más $50 \%(\lambda>.70)$ de su variabilidad (Ver tabla 4). En lo que corresponde al factor AR (Ver tabla 4), las cargas factoriales alcanzaron valores desde .42 (ítem 22 "Me canso rápidamente") hasta .82 (ítem 21 "Me siento Bien" e ítem 33 "Me siento seguro (a)"). Pero, se exceptúan de este rango los ítems 31 "Tomo las cosas muy a pecho", 34 "Procuro evitar enfrentarme a las crisis y dificultades" y 39 "Soy una persona estable", pues las cargas factoriales obtenidas fueron respectivamente de $.07, .24$ y .08, por debajo del punto de referencia que acepta valores que expliquen, mínimamente, el $10 \%$ de la variabilidad de los ítems $(\lambda>.30)$, aun cuando el ítem 34 podría ser justificado a través del criterio de significancia estadística $(\lambda>.20$, Morales, 2013).

A razón de tal evidencia, se restructuro el modelo a una versión de 37 ítems en las mismas dimensiones: AE y AR, esta última con solamente 17 ítems, ya que se eliminaron los ítems 31, 34 y 39 cuyas cargas factoriales no obtuvieron valores mínimamente esperados en el modelo 1. Esta restructuración (modelo 2) reportó mínimos cambios en los valores de ajuste $\left(\mathrm{x}^{2} / \mathrm{gl}=1.74\right.$; $\mathrm{RSMEA}=.080$ (IC 95\% $=.072-.088) ; \mathrm{SRMR}=.096 ; \mathrm{CFI}=.988$ y TLI $=.987$; Ver tabla 3). Así como, en 
las cargas factoriales (Ver tabla 4); la dimensión AE presentó valores desde .71 a .96 y la dimensión AR desde .42 a .91. Al igual que en la valoración del ajuste, las variaciones con respecto al modelo 1 fueron mínimas. Tomando en cuenta estos datos, se podría entender que, el modelo 2 presenta mejores valores; pero con mínimas diferencias.

\section{Tabla 4}

Cargas factoriales y fiabilidad

\begin{tabular}{|c|c|c|c|c|c|}
\hline \multirow{2}{*}{ Factor / ítem } & \multicolumn{2}{|c|}{ Ansiedad estado $(\lambda)$} & \multirow{2}{*}{ Factor / ítem } & \multicolumn{2}{|c|}{ Ansiedad rasgo $(\lambda)$} \\
\hline & M1 & M2 & & M1 & M2 \\
\hline 1 & 0.83 & 0.83 & 21 & 0.85 & 0.85 \\
\hline 2 & 0.79 & 0.79 & 22 & 0.42 & 0.42 \\
\hline 3 & 0.71 & 0.71 & 23 & 0.78 & 0.78 \\
\hline 4 & 0.75 & 0.75 & 24 & 0.65 & 0.65 \\
\hline 5 & 0.87 & 0.87 & 25 & 0.61 & 0.61 \\
\hline 6 & 0.81 & 0.81 & 26 & 0.44 & 0.44 \\
\hline 7 & 0.75 & 0.75 & 27 & 0.54 & 0.55 \\
\hline 8 & 0.84 & 0.84 & 28 & 0.80 & 0.79 \\
\hline 9 & 0.82 & 0.82 & 29 & 0.72 & 0.71 \\
\hline 10 & 0.88 & 0.88 & 30 & 0.76 & 0.76 \\
\hline 11 & 0.74 & 0.74 & 31 & 0.07 & - \\
\hline 12 & 0.82 & 0.82 & 32 & 0.80 & 0.79 \\
\hline 13 & 0.70 & 0.71 & 33 & 0.85 & 0.84 \\
\hline 14 & 0.85 & 0.85 & 34 & 0.24 & - \\
\hline 15 & 0.72 & 0.72 & 35 & 0.76 & 0.76 \\
\hline 16 & 0.77 & 0.77 & 36 & 0.66 & 0.67 \\
\hline 17 & 0.76 & 0.76 & 37 & 0.73 & 0.73 \\
\hline 18 & 0.77 & 0.77 & 38 & 0.66 & 0.66 \\
\hline 19 & 0.83 & 0.83 & 39 & 0.08 & - \\
\hline 20 & 0.90 & 0.90 & 40 & 0.50 & 0.50 \\
\hline$\omega$ & 0.96 & 0.96 & $\omega$ & 0.90 & 0.91 \\
\hline
\end{tabular}

Nota: M1: Modelo original 40 ítems; M2: Modelo restructurado con 37 ítems; $\lambda$ : Carga factorial; $\omega$ : Coeficiente de confiabilidad omega.

Aún con las cargas factoriales por debajo de los parámetros de aceptabilidad en tres de los 20 ítems del factor AR, el reporte de la confiabilidad (Ver tabla 4) evidenció valores deseables para este factor $(\omega=.90)$, sucediendo de igual manera en el factor AE, el valor obtenido demostró su consistencia $(\omega=.96)$. En el segundo modelo, en el cual se eliminan los tres ítems con carga factorial no aceptable en la dimensión AR, se advirtió un leve aumento de $(\omega=.91$; Ver tabla 4$)$. 


\section{DISCUSIÓN}

Los resultados obtenidos en la validación del IDARE en adultos de la ciudad de Trujillo arrojaron evidencias de validez de contenido, evidencias de validez de estructura y la confiabilidad del instrumento, las cuales se discutirán a continuación.

En las evidencias de validez de contenido se identificó unanimidad en la aceptación de que los ítems que conforman la escala de Ansiedad-Estado responden a la medida de este constructo, de acuerdo a la revisión de los expertos. Los valores obtenidos por los ítems al aplicar la fórmula de Aiken, reportaron ser aceptables a la perspectiva de la estadística inferencial estipulada en el criterio de Escurra (1988). Según este criterio, los valores de Aiken obtenidos en un punto de referencia de .77 son estadísticamente significativos $(\mathrm{p}<.05)$ cuando se recopila información de 9 jueces. En referencia a ello, los valores de claridad obtenidos fueron de .81 en adelante; de igual manera en coherencia; $y$, en relevancia de .78 a .96; por lo tanto, se estaría comprobando, de acuerdo a la evidencia empírica, la aceptación de validez por los ítems que forman parte de esta escala. Esta validez se traduce en la redacción clara de los ítems, pero, a la vez son coherentes en la medición de condiciones emocionales transitorias del organismo humano que varía en intensidad y fluctúa con el tiempo; y se entiende que resulta de la situación o circunstancia del momento (García-Batista, et al., 2015).

En cuanto a la escala Ansiedad-Rasgo tres ítems de los veinte fueron observados, ya que los valores obtenidos en los criterios de coherencia y relevancia -como se verá en seguida- no superaron el punto de corte de referencia supuesto por Escurra (1988). En coherencia fueron los ítems 33, "Me siento seguro (a)" y 36, "Soy una persona estable". Este criterio advierte de la lógica que debe tener el ítem con el indicador y, en su defecto, con el constructo medido; en este caso, la Ansiedad-Rasgo. Este constructo comprende las respuestas afectivas ansiosas surgidas independientemente del momento, pero influenciadas por aspectos de la personalidad del propio sujeto (García-Batista et al., 2015). En función de tal conceptualización, el ítem 33, "Me siento seguro (a)" demostraría ser una expresión de una respuesta afectiva, siendo tal respuesta la inseguridad; la cual, también ha sido considerada como una expresión emocional (Farabaugh et al., 2005). Por su parte, el ítem 36, "Me siento satisfecho (a)", también demostraría ser parte de una respuesta afectiva (Montaño et al., 2009).

Según los análisis previos, se revisó la literatura en torno a la validez de contenido aplicando la fórmula de Aiken. Al respecto, se encontró que estos pueden ser valorados también a partir de sus intervalos de confianza (Merino \& Libia, 2009). Consecuentemente, al estimarlos y referir lo señalado por Cicchetti (1994) en un criterio conservador; los 3 ítems (33, 36 y 39) estarían dentro del estándar aceptable para advertir de su cumplimiento de validez. Entonces, sobre la evidencia empírica y la revisión del fraseo de los ítems se puede respaldar su validez, sin 
que ello restrinja la posibilidad de analizar en estudios consecuentes su comportamiento sobre otras fuentes de evidencia de contenido.

En correspondencia a los hallazgos sobre la validez basada en la estructura, la evidencia obtenida llevó a respaldar la idoneidad de la estructura en base a dos modelos de dos dimensiones: AE y AR; el primero de 40 ítems y el segundo de 37 ítems. Los valores identificados en el ajuste demostraron superar los estándares que se sugieren para aceptar la hipótesis de correspondencia entre la matriz hipotética y la matriz reproducida o lo que es, para el caso de la investigación, el ajuste entre el modelo hipotético de dos dimensiones y los datos que se obtuvieron de los adultos de Trujillo. Los criterios de referencia fueron tomados de $\mathrm{Hu}$ y Bentler (1999). Con esto se entiende, desde un plano general, que la medición de la ansiedad, desde el planteamiento conceptual de Spielberger (1975) la ansiedad puede ser comprendida a partir de dos perspectivas: la ansiedad como estado y la ansiedad como rasgo; estaría siendo representado de manera adecuada en el IDA$\mathrm{RE}$, para obtener valoraciones que lleven a su comprensión.

Estos hallazgos generales no limitan su observación en un plano especifico. La carencia de pertenencia o representatividad por parte de tres de los veinte ítems de la dimensión Ansiedad-Estado: el ítem 31 "Tomo las cosas muy a pecho", 34 "Procuro evitar enfrentarme a las crisis y dificultades" y el ítem 39 "Soy una persona estable".

Los hallazgos de esta investigación guardan similitud con respecto a los estudios de Rojas-Carrasco (2010) y Espíritu (2018) respecto a la no pertinencia de ítem 34; tales estudios, también reportaron a este enunciado con una carga factorial baja. Y, es importante precisar la diferencia de métodos utilizados con respecto a Carrasco (2010), quién utilizó análisis de factores por medio de la exploración; mientras que, con Espíritu (2018) la diferencia responde a los índices de ajuste elegidos, siendo menos estudiados, los elegidos en el estudio citado. Sin embargo, independiente de tales diferencias, se reporta con todos los hallazgos un comportamiento persistente en no representatividad por parte del ítem 34 .

Con respecto a los ítems 31 y 39 solamente se encontró evidencia que cuestiona su pertinencia como indicador de medida de la AR, en esta investigación. Por lo que, dada la falta de evidencia en este ítem, como se comprueba al observar también los estudios citados (Espíritu, 2018; Rojas-Carrasco, 2010), se advierte la necesidad de realizar nuevas investigaciones en las que se concentre especial interés en estos ítems, de tal manera que se pueda responder a los vacíos acá identificados.

En este sentido, la restructuración del IDARE en un modelo de dos dimensiones, pero con solamente 17 ítems para la escala de ansiedad -rasgo, al obtener índices de bondad de ajuste favorables. Entendiendo que, a la espera de nuevas investigaciones que repliquen el propósito de validación en muestras de mayor 
tamaño y diferentes características, se pueda utilizar el IDARE en adultos de Trujillo. En tanto se logra entender el comportamiento de los ítems tal población.

Por otro lado, al analizar la fiabilidad la dimensión AR evidencio valor dentro de los estándares de aceptación (Campo-Arias \& Oviedo, 2008), mientras que, la dimensión AE se reportó un valor por sobre los estándares (sobre .90), lo cual, es indicador de redundancia en la medición del constructo (AE), pero, sin llegar a señalar que la dimensión sea inconsistente o no confiable. En la restructuración del modelo (modelo 2), la dimensión que perdió ítems (AR), demostró un leve aumento de su valor de consistencia, sin generar marcadas diferencias. Tomando en cuenta ello, es posible señalar que el IDARE, en esta investigación, como también en los estudios previos a este (Espíritu, 2018; Rojas-Carrasco, 2010), demuestran garantías científicas para su uso como medida de la ansiedad.

Finalmente, es importante hacer presente algunas limitaciones a las que ve se enfrentó esta investigación. La primera referida a un tamaño representativo de la muestra a la cual no fue posible acceder, debido a las circunstancias de pandemia que vive la sociedad actual y a la no participación activa de los padres a pesar de utilizar los medios comúnmente utilizados (redes sociales) para su difusión. La segunda, que se desprende de la anterior, refiere al acceso no presencial a los participantes, lo cual impidió dar las indicaciones de aplicación de manera estandarizada y monitorizada y resolver las dudas que puedan surgir en el proceso que el participante resuelve la escala; debido a la aplicación virtualizada por medio de formularios online, que llegaron a los participantes a través de redes sociales o correo electrónico. La Tercera, la falta de datos (estudios previos) en cuanto a validaciones del IDARE en el contexto y población estudiada, lo cual por un lado, impiden llegar a generalizaciones mayores y contraste directo de hallazgos con contextos similares y por ende el alcance de la aplicación del test validado, pero, por otro lado, permite identificar nuevas brechas para ampliar los estudios de validación.

\section{CONCLUSIONES}

Del análisis de los resultados, se arriba a las conclusiones siguientes: por un lado, el IDARE demuestra estar compuesto por ítems claros, coherentes y relevantes para la medición de la ansiedad en su modalidad de estado y su modalidad de rasgo. Lo cual demostraría el cumplimiento de la validez de contenido, de acuerdo con la revisión de expertos.

Por otro lado, la estructura hipotética de 2 grandes dimensiones del IDARE Ansiedad-Estado y Ansiedad-Rasgo (este último con 17 ítems), demostraría ser de utilidad para obtener una valoración sobre la ansiedad en población adulta de Trujillo. 
Finalmente, el IDARE demostró también ser consistente o mostrar confiabilidad al valorarse la ansiedad por medio de cada una de sus escalas.

\section{Aspectos éticos}

La autora declara no haber incurrido en aspectos antiéticos respetando los códigos de investigación con humanos reseñados en el código de Ética profesional del Colegio de psicólogos del Perú.

Se solicitó la autorización de cada participante a través de la aceptación del consentimiento informado el cual brindaba datos de la naturaleza, medios y objetivos de la investigación.

Para fines de adaptación, validez y confiabilidad del instrumento se solicitó la autorización mediante correo electrónico a la editorial El Manual Moderno, donde se recibió una respuesta positiva, por lo cual se procedió con los objetivos de la investigación.

\section{Conflicto de intereses}

La autora declara no haber presentado conflicto de intereses al realizar la investigación y articulo.

\section{REFERENCIAS}

Ato, M., López, J. y Benavente, A. (2013). Un sistema de clasificación de los diseños de investigación en psicología. Anales de psicología, 29 (3), 1038-1059. http://dx.doi. org/10.6018/analesps.29.3.178511

Bouayed, J., Rammal, H. \& Soulimani, R. (2009). Oxidative stress and anxiety. Oxidative Medicine and Cellular Longevity, 2(2), 63-67. doi:10.4161/oxim.2.2.7944

Broca, P. (2019). Depresión y ansiedad: esto debemos saber los peruanos de nuestra salud mental. El comercio. https://elcomercio.pe/somos/historias/depresion-ansiedad-estodebemos-peruanos-nuestra-salud-mental-noticia-ecpm-628100-noticia/?ref=ecr

Brown, T. A. (2006). Confirmatory factor analysis for applied research. The Guilford Press.

Browne, M. y Cudeck, R. (1993). Alternative ways of assessing model fit. In K. A. Bollen y J. S. Long (Eds.), Testing structural equation models (pp. 136-162). Sage.

Buela-Casal, G., Guillén-Riquelme, A. \& Seisdedos, N. (2011). STAI: Cuestionario de ansiedad estado-rasgo. Adaptación española. Madrid: TEA Ediciones.

Campo-Arias, A., \& Oviedo, H. C. (2008). Propiedades psicométricas de una escala: la consistencia interna. Revista Salud Pública, 10 (5), 831-839. https://www.redalyc.org/ $\mathrm{pdf} / 422 / 42210515 . \mathrm{pdf}$

Charter, R. A. (2003). A breakdown of reliability coefficients by test type and reliability method, and the clinical implications of low reliability. Journal of General Psychology, 130 (3), 290-304. https://doi.org/10.1080/00221300309601160 
Cicchetti, D. (1994) Guidelines, criteria, and rules of thumb for evaluat-ing normed and standardized assessment instruments in psychology. Psychological Assesments, 6, 284-290. https://doi.org/10.1037/1040-3590.6.4.284

Díaz, J. (2018). El 22\% de la población ha sufrido ansiedad y depresión. La República. https://larepublica.pe/sociedad/1336981-22-poblacion-sufrido-ansiedad-depresion/

Escurra, L. (1988). Cuantificación de la validez de contenido por criterio de jueces. Revista de psicología, 6 (1-2), 103 - 111. http://revistas.pucp.edu.pe/index.php/psicologia/ article/view/4555

Espíritu, Y. (2018). Propiedades psicométricas de la escala de Ansiedad Estado - Rasgo en universitarios de la ciudad de Chimbote. [Tesis de pregrado]). Universidad Privada Cesar Vallejo, Nuevo Chimbote, Perú. https://hdl.handle.net/20.500.12692/26297

Farabaugh, A., Fava, M., Mischoulon, D., Sklarsky, K. \& Petersen, T. (2005). Relationships between major depressive disorder and comorbid anxiety and personality disorders. Comprehensive Psychiatry, 46, 266-271. https://doi.org/10.1016/j.comppsych.2004.10.005

García-Batista, Z., Cano-Vindel, A. \& Herrera-Martínez, S. (2015). Propiedades psicométricas del inventario de situaciones y respuestas de ansiedad (ISRA) en una muestra dominicana. Pensamiento Americano, 8(15), 56-66. doi:10.21803/penamer.8.15.326

García-Batista, Z., Cano-Vindel, A. \& Herrera-Martínez, S. (2017). Propiedades psicométricas del Inventario de ansiedad estado-rasgo en población general y hospitalaria de República Dominicana. Ansiedad y Estrés, 23 (2), 53 - 58. doi: 10.1016/j. anyes.2017.09.004

García, M. (2011). Análisis causal con ecuaciones estructurales de la satisfacción ciudadana con los servicios municipales [Trabajo de master, Programa de máster en técnicas estadísticas, Universidad de Santiago de Compostela]. http://eio.usc.es/pub/ $\mathrm{mte} /$ descargas/proyectosfinmaster/proyecto_610.pdf

Guevara-Cordero, C., Rodas-Vera, N., Varas-Loli, R. (2019). Relación entre autoconcepto y ansiedad estado-rasgo en universitarios peruanos. Revista de Investigación enPsicología UNMSM, Vol. 22 - N. 2 - 2019, pp. 251 - 264. doi: 10.15381/rinvp.v22i2.17425

Guillen-Riquelme, A. \& Buela-Casal, G. (2015). Estructura factorial del cuestionario de Ansiedad Estado-Rasgo (STAI) para pacientes diagnosticados con depresión. Salud Mental, 38(4), 293-298. doi: 10.17711/sm.0185-3325.2015.040

Heckathorn, D. D. (2007). Extensions of Respondent-Driven Sampling: Analyzing Continuous Variables and Controlling for Differential Recruitment. Sociological Methodology, 37 (1), 151-208. https://doi.org/10.1111/j.1467-9531.2007.00188.x

$\mathrm{Hu}$, L. y Bentler, P. (1999). Cutoff Criteria for Fit Indexes in Covariance Structure Analysis: Conventional Criteria Versus. Structural Equation Modeling: A Multidisciplinary Journal New Alternatives, 6 (1), 1 - 55. http://dx.doi. org/10.1080/10705519909540118 
Hu, L. y Bentler, P. (1995). Evaluating model fit. In R. H. Hoyle (Ed.), Structural equation modeling (pp. 76-99). Sage.

Instituto Nacional de Salud Mental (2013). Estudio Epidemiológico de Salud Mentalen Lima Metropolitana y Callao - Replicación 2012. Anales de Salud Mental, 1 (29). https://www.insm.gob.pe/investigacion/archivos/estudios/2012\%20ASM\%20 -EESM\%20-LM.pdf

Merino, C. y Libia, J. (2009). Intervalos de confianza asimétricos para el índice la validez de contenido: Un programa Visual Basic para la V de Aiken. Anales de psicología, 25 (1), 169-171. https://dialnet.unirioja.es/servlet/articulo?codigo=2973711

Morales, P. (2013). El Análisis Factorial en la construcción e interpretación de tests, escalas y cuestionarios. http://www.upcomillas.es/personal/peter/investigacion/AnalisisFactorial.pdf

Montaño, M., Palacios, J. y Gantiva, C. (2009). Teorías de la personalidad. Un análisis histórico del concepto y su medición Psychologia. Avances de la disciplina, 3 (2), 81-107. https://www.redalyc.org/pdf/2972/297225531007.pdf

Organización Mundial de la Salud (2019). Salud mental en el lugar de trabajo. (mayo del 2019). https://www.who.int/mental_health/in_the_workplace/es/

Organización Mundial de la Salud (2020). La inversión en el tratamiento de la depresión y la ansiedad tiene un rendimiento del 400\%. (20 de marzo del 2020). https://www. who.int/mediacentre/news/releases/2016/depression-anxiety-treatement/es/

Rivera, N. (2020). Evidencia de los procesos psicométricos del Inventario de la ansiedad rasgo-estado (IDARE) en escolares de 4to y 5to de secundaria de Instituciones Educativas de la ciudad Piura (Tesis de pregrado). Universidad Privada Cesar Vallejo, Piura, Perú. https://hdl.handle.net/20.500.12692/42868

Rojas, E. (2014). Cómo superar la ansiedad: La obra definitiva para vencer el estrés, las fobias y las obsesiones. Barcelona: Planeta.

Rojas-Carrasco, K. (2010). Validación del Inventario de Ansiedad Rasgo-Estado en padres con un hijo en terapia intensiva. Revista Médica del Instituto Mexicano del Seguro Social, 491- 496. https://www.redalyc.org/pdf/4577/457745510005.pdf

Silva, C., Hernández, A., Jiménez. B., Alvarado, N. (21016). Revisión de la estructura interna de la subescala de rasgo del Inventario de Ansiedad Rasgo-Estado para jóvenes de habla hispana. Psicología y Salud, Vol. 26 (2), 253-262. https://psicologiaysalud. uv.mx/index.php/psicysalud/article/viewFile/2201/3937

Spielberger, C. D., Gorsuch, R. L. \& Lushene, R. E. (1970). Manual for the State-TraitAnxiety Inventory. Palo Alto, CA: Consulting Psychologists Press.

Spielberger, C., \& Díaz- Guerrero, R. (1975). IDARE Inventario de ansiedad: rasgo estado - manual e instructivo. México: Manual moderno.

Ventura-León, J. (2017). ¿Población o muestra?: Una diferencia necesaria. Revista Cubana de Salud Pública, 43 (4). http://www.revsaludpublica.sld.cu/index.php/spu/article/ view/906/948 
Vera, C. (2018). Ansiedad rasgo-estado y estilo personal del terapeuta en psicólogos que trabajan con pacientes oncológicos en lima metropolitana (Tesis de pregrado). Pontificia Universidad Católica del Perú. http://hdl.handle.net/20.500.12404/13081

Zych, I. (2011). Cómo sobreponerse a la ansiedad. Un manual práctico para que la ansiedad deje de controlar tu vida. Madrid: Pirámide. 